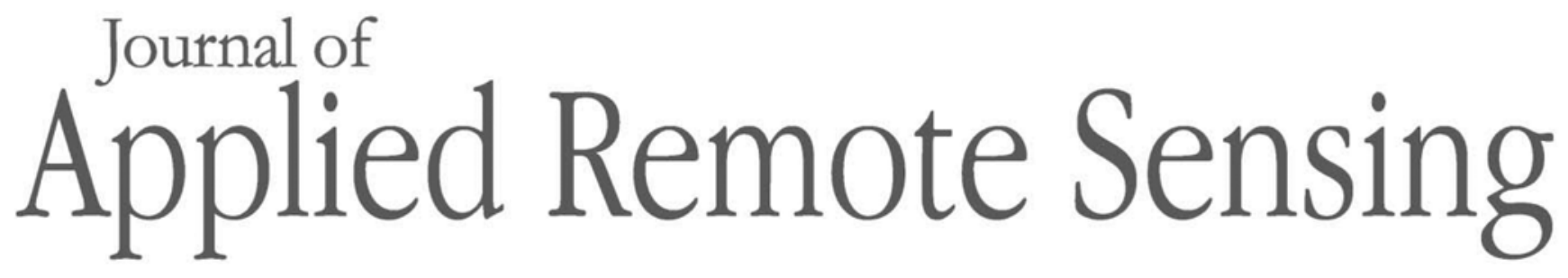

RemoteSensing.SPIEDigitalLibrary.org

\title{
Vicarious radiometric calibration/ validation of Landsat-8 operational land imager using a ground reflected radiance-based approach with Baotou site in China
}

\author{
Yaokai Liu \\ Lingling Ma \\ Ning Wang \\ Yonggang Qian \\ Shi Qiu \\ Chuanrong Li \\ Lingli Tang
}

Yaokai Liu, Lingling Ma, Ning Wang, Yonggang Qian, Shi Qiu, Chuanrong Li, Lingli Tang, "Vicarious radiometric calibration/validation of Landsat- 8 operational land imager using a ground reflected 


\title{
Vicarious radiometric calibration/validation of Landsat-8 operational land imager using a ground reflected radiance-based approach with Baotou site in China
}

\author{
Yaokai Liu, ${ }^{\text {a,b }}$ Lingling Ma, ${ }^{\mathrm{a}, *}$ Ning Wang, ${ }^{\mathrm{a}}$ Yonggang Qian, ${ }^{\mathrm{a}}$ \\ Shi Qiu, ${ }^{a}$ Chuanrong $\mathrm{Li}^{\mathrm{a}},{ }^{\text {and Lingli Tang }}{ }^{\mathrm{a}}$ \\ ${ }^{a}$ Chinese Academy of Sciences, Academy of Opto-Electronics, Key Laboratory of \\ Quantitative Remote Sensing Information Technology, Beijing, China \\ ${ }^{b}$ University of Chinese Academy of Sciences, Beijing, China
}

\begin{abstract}
Radiometric calibration of the Landsat-8 operational land imager (OLI) sensor is a key step that enables additional quantitative research and application of the data. This study introduces a ground reflected radiance-based approach to vicarious radiometric validate the Landsat-8 OLI onboard radiometric calibration results. Vicarious radiometric calibration/ validation field campaign of the Landsat- 8 OLI sensor was carried out at the Baotou site in Inner Mongolia, China, on March 27, 2015, October 28, 2015, March 29, 2016, and April 21,2016 . The relative errors of the OLI bands are within $5 \%$ for the ground reflected radiance-based approach when compared with satellite observations of top of atmosphere (TOA) radiance in the OLI bands, and the average $1 \sigma$ standard deviation of the four results using the vicarious radiometric calibration is less than 0.025. A well-calibrated Terra MODIS sensor was used to cross-validate the Landsat-8 OLI sensor, and the relative errors are within $10 \%$. The results suggest that the OLI onboard the Landsat- 8 satellite displays stable radiometric performance for the four calibration/validation days. An uncertainty analysis of the ground reflected radiance-based approach showed an overall uncertainty of less than $4.5 \%$, taking into account uncertainty sources, including surface characteristics, atmospheric characteristics, radiative transfer, solar irradiance, and the calibration model. (c) The Authors. Published by SPIE under a Creative Commons Attribution 3.0 Unported License. Distribution or reproduction of this work in whole or in part requires full attribution of the original publication, including its DOI. [DOI: 10 .1117/1.JRS.11.044004]
\end{abstract}

Keywords: vicarious radiometric calibration/validation; ground reflected radiance-based approach; Landsat-8 operational land imager; uncertainty analysis; calibration site.

Paper 170682 received Jul. 31, 2017; accepted for publication Nov. 7, 2017; published online Dec. 15, 2017.

\section{Introduction}

So far, eight Landsat series satellites, including Landsat-1 to Landsat-8, have been launched since the first Landsat satellite launched successfully on July 23, 1972. Landsat-8, which was launched on February 11, 2013, is the latest satellite in the Landsat series. ${ }^{1}$ Images with 30-m spatial resolution have been acquired since Landsat- 4 was launched in 1984. Also, the Landsat-4 to Landsat- 8 satellite orbital repeating cycle is every 16 days. Besides that, the repeating cycle of Landsat 1 to 3 sensors was every 18 days. Unlike prior Landsat instruments in which the visible and near-infrared (VNIR) and thermal images were acquired with the same sensor, there are two instruments onboard the Landsat- 8 platform. These instruments are the operational land imager (OLI), which has nine VNIR multispectral channels covering a spectral range of 0.4 to $2.5 \mu \mathrm{m}$, and the thermal infrared sensor, which has two thermal channels that cover a spectral

*Address all correspondence to: Lingling Ma, E-mail: aoe_pi@163.com 
range of 10.6 to $12.5 \mu \mathrm{m} .^{2}$ As one of the Landsat series satellites, the instrument payload on the Landsat-8 platform has characteristics that are similar to those of prior Landsat sensors in terms of their swath width and spectral bandwidths, as well as their radiometric and geometric accuracy. ${ }^{3,4}$

Due to their temporal, spatial, spectral, and radiometric characteristics, the remote sensing data acquired by the Landsat instruments over the long-term provide us with an important way to understand both local and global environment change. ${ }^{1}$ For example, thematic forest classifications derived from Landsat data have been used to assess forest cover density and productivity. ${ }^{5}$ Landsat remote sensing data have also been widely used to retrieve vegetation biophysical characteristics, such as leaf area index values, canopy cover, and biomass, based on solar reflective spectrum information. ${ }^{6,7}$ Landsat images have also been used to monitor the seasonal and interannual dynamics of ecosystems. For example, Dymond et al. ${ }^{5}$ used interannual Landsat data to assess conifer forest structure by mapping hardwood and mixed forests at the species level.

However, many applications, such as the assessment of human activities on land surface ecology and vegetation monitoring, depend on the long-term stability of satellite radiometric calibrations to produce useful estimates of long-term variability. ${ }^{8}$ In addition, the ability to detect land cover change and perform land use classifications with Landsat images depends on accurate radiometric characterization and calibration of Landsat instruments. ${ }^{9}$ Further applications, such as the inversion of terrestrial variables and biophysical parameters, based on Landsat images also require the assessment of radiometric consistency to ensure interimage comparability, due to the radiometric differences between Landsat series images. ${ }^{10}$ Therefore, absolute radiometric calibration performed on Landsat satellite images is a critical step in the retrieval of land, atmosphere, and ocean information. Generally, absolute radiometric calibration enables the conversion of the digital numbers that make up an image to values with physical units at the sensor's spectral radiance. ${ }^{11}$

During the past several years, many methods have been developed to provide radiometric calibrations of the Landsat series instruments, and these methods generally can be summarized as prelaunch and laboratory-based, postlaunch and onboard-based, or postlaunch and vicarious radiometric calibrations. ${ }^{12}$ Prelaunch radiometric calibrations are usually carried out in the laboratory using spherical integrating sources. Unfortunately, the radiometric and spectral performance of sensors has usually degraded over time after the Landsat satellites were launched into space, due to the change in environment and aging of the sensors. Moreover, this process may lead to situations in which the observed data cannot be radiometrically calibrated using the prelaunch calibration coefficients. To overcome this problem, postlaunch onboard-based methods that use calibrator sources, such as the sun, lamps, and blackbodies are used to radiometrically calibrate the Landsat sensors in flight. ${ }^{13-19}$ However, on-board calibration also has some drawbacks. For example, onboard calibration cannot provide a calibration that is of higher accuracy than the laboratory calibration of the onboard calibrator. ${ }^{13}$ Also, the onboard radiometric calibrators have been reported to have limitations in terms of their ability to track the instruments' radiometric stability, although the solar diffuser showed the best performance. ${ }^{18}$ Moreover, the absolute radiometric calibration accuracy of onboard calibration may be worse than that of the prelaunch calibration, due to the aging and increasing uncertainty associated with the onboard calibrators over time. ${ }^{13}$

Vicarious radiometric calibration provides a useful method for monitoring the radiometric performance of the onboard satellite sensors. Vicarious radiometric calibration is one of the most used methods to evaluate the radiometric calibration performance of Landsat series sensors using in situ measurements over pseudoinvariant calibration sites at the time of satellite overpass. ${ }^{11}$ Vicarious radiometric calibration can be used as an assistant calibration way of the onboard calibrators, and it can also permit a traceable understanding of the radiometric stability of the onboard sensor, although it has the drawbacks of low-frequency data collection and is expensive in terms of personnel time and cost. ${ }^{11,13}$ Many vicarious radiometric calibration methods have been developed, and these methods can be divided into groups, such as reflectance-based, airborne radiance-based, and irradiance-based calibration methods. Both the reflectance-based and radiance-based approaches predict the at-sensor radiance using radiative transfer model calculations, constrained by the measured surface reflectance or radiance and atmospheric 
conditions..$^{20,21}$ However, the irradiance-based approach needs to measure the atmospheric characteristics as well as the diffuse-to-global irradiance, which is the ratio between measured sky irradiance and total irradiance including solar irradiance and sky irradiance. ${ }^{22}$

This paper aims to assess the radiometric performance of the in-orbit OLI onboard Landsat-8 by comparing onboard radiometrically calibrated OLI band top of atmosphere (TOA) radiance values with those predicted using a ground reflected radiance-based vicarious radiometric calibration approach. To achieve this purpose, four vicarious radiometric calibration/validation field campaigns were carried out at the Baotou site in China and applied to the OLI radiometric validation. This paper is organized as follows. Section 2 describes the Baotou site and the datasets that have been used to calibrate the OLI. Section 3 describes the theory underlying the ground reflected radiance-based approach. Section 4 describes the OLI radiometrically validation results by using the ground reflected radiance-based approach. An uncertainty analysis is also given in Sec. 4. Finally, conclusions on the Landsat-8 OLI radiometrically validation with ground reflected radiance-based approach have been drawn in Sec. 5 .

\section{Calibration and Validation Site and Datasets}

\subsection{Calibration and Validation Site Description}

It is very important to select a calibration and validation (Cal\&Val) site for vicarious radiometric calibration/validation when using reflectance-based, irradiance-based, or radiance-based approaches. An ideal Cal\&Val site should typically have several characteristics summarized by Thome. ${ }^{23}$ In this study, the Baotou site in China is selected as our vicarious radiometric calibration site. The Baotou site has been established to assess the radiometric and spectral performance of the high spatial resolution sensor payloads on airborne and satellite platforms with support from the National High Technology Research and Development Program of China. The coordinates of the Baotou site are $40.88^{\circ} \mathrm{N}, 109.53^{\circ} \mathrm{E}$. This site is located at Urad Qianqi in the Inner Mongolia of north China, and it has an average elevation of $\sim 1270-\mathrm{m}$ above the sea level. The semidesert, clear sky, and uniform surface of Urad Qianqi are considered to be ideal for a calibration experiment.

There are two categories of targets at the Baotou site for vicarious radiometric calibration. One category includes the permanent artificial targets, which are suitable for air-borne and highspatial resolution sensors. The other is the large area of desert targeted by moderate spatial resolution, such as Landsat series satellite optical sensors. In this study, the large area of desert target will be used to radiometrically calibrate the OLI sensor. The size of the desert target is large enough to ensure that no less than $10 \times 10$ pixels area of OLI multispectral band will be captured with a 30-m spatial resolution. Figure 1 shows pictures of permanent artificial and desert targets at the Baotou site. In addition, Fig. 2 shows a subset of an image of the Baotou site acquired by the OLI multispectral sensor on March 29, 2016. The red box indicates the position of the desert target, and the green box indicates the position of the artificial targets. The distance between the desert target and the artificial targets is $\sim 2 \mathrm{~km}$.

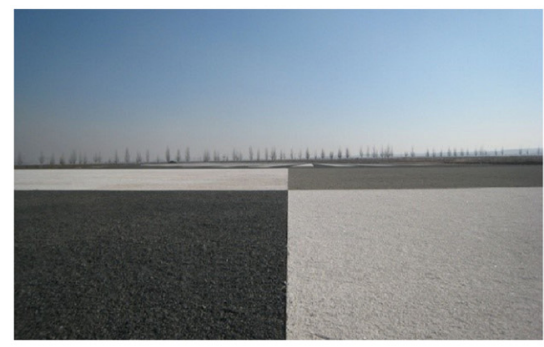

(a)

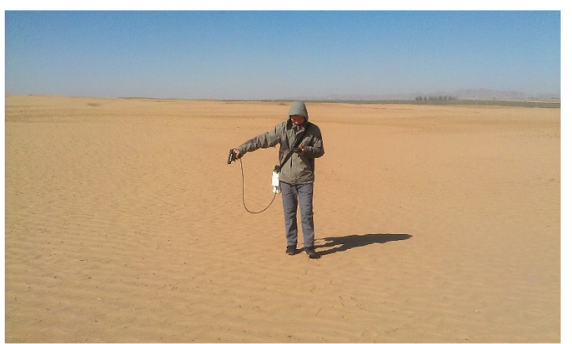

(b)

Fig. 1 Pictures of the (a) permanent artificial targets and (b) the desert target at the Baotou site, Inner Mongolia, China. 


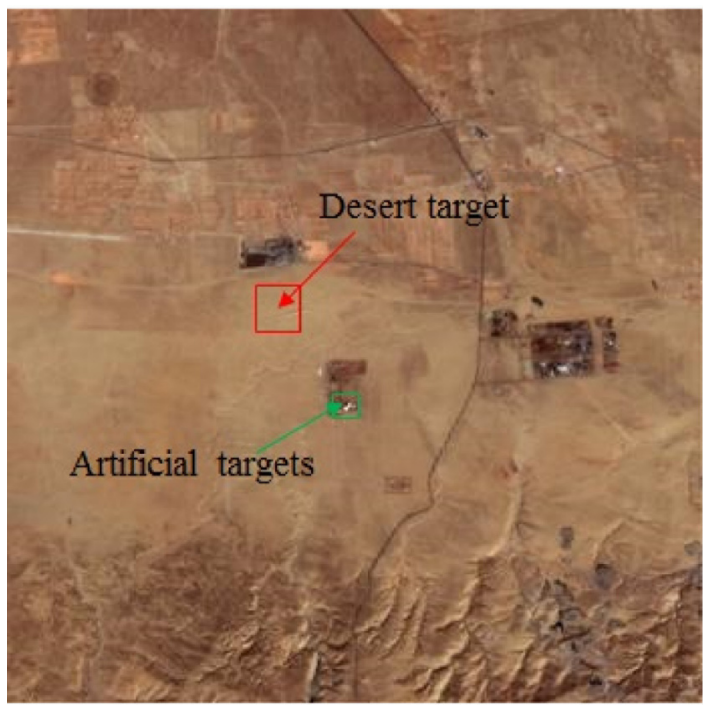

Fig. 2 A subset RGB image of the Baotou site obtained by Landsat-8 OLI on March 29, 2016 (the red box indicates the position of the desert target, and the green box indicates the position of the artificial targets).

Table 1 Specifications of the center wavelength, band coverage, and resolution associated with the Landsat OLI bands.

\begin{tabular}{lccccccccc}
\hline \hline Channels & 1 & 2 & 3 & 4 & 5 & 6 & 7 & 8 & 9 \\
\hline Center wavelength (nm) & 442.96 & 482.04 & 561.41 & 654.59 & 864.67 & 1608.86 & 2200.73 & 589.50 & 1373.43 \\
Band coverage (nm) & $427-$ & $436-$ & $513-$ & $626-$ & $830-$ & $1516-$ & 20382356 & $488-$ & $1341-$ \\
& 459 & 528 & 611 & 692 & 901 & 1699 & & 693 & 1410 \\
Resolution (m) & 30 & 30 & 30 & 30 & 30 & 30 & 30 & 15 & 30 \\
Description & $\begin{array}{l}\text { Coastal } \\
\text { aerosol }\end{array}$ & Blue & Green & Red & NIR & SWIR_1 & SWIR_2 & Pan & Cirrus \\
\hline \hline
\end{tabular}

\subsection{Landsat-8 OLI Datasets}

The OLI sensor onboard the Landsat-8 satellite includes nine channels, and the specifications for the center wavelength, band coverage, and resolution are shown in Table $1 .{ }^{1}$ The designed revisiting period of the Landsat- 8 satellite is 16 days, and the overpass time at the Baotou site is approximately 11:30 am Beijing local time. However, there are two path/row combinations (namely, 127/32 and 128/32) that cover the Baotou site. Therefore, the Baotou site can, in principle, be observed by the Landsat- 8 OLI sensor every 8 days. However, only four clear scenes were successfully acquired at the same time as ground measurements; these paired measurements occurred on March 27, 2015, October 28, 2015, March 29, 2016, and April 21, 2016. The collected L1T level images were radiometric calibrated with radiometric calibration coefficients including gains and bias provided with L1T products. OLI calibration gains were determined prelaunch in the laboratory and using onboard calibrators to monitor their trends. While OLI calibration bias is determined from the calibration shutter acquired prelaunch and postlaunch imaging of every path. Then, the mean onboard radiometric calibrated OLI band TOA radiance over the desert target at the Baotou site was extracted from the radiometrically calibrated images with radiance multiplicative and additive factors from metadata files provided with L1T products. Figure 3 shows the averaged and $1 \delta$ standard deviation calculated from the selected 100 pixels' OLI band TOA radiance of the desert target. The low $1 \delta$ standard deviation suggests that our selected desert target has very good uniformity and is suitable for vicarious 


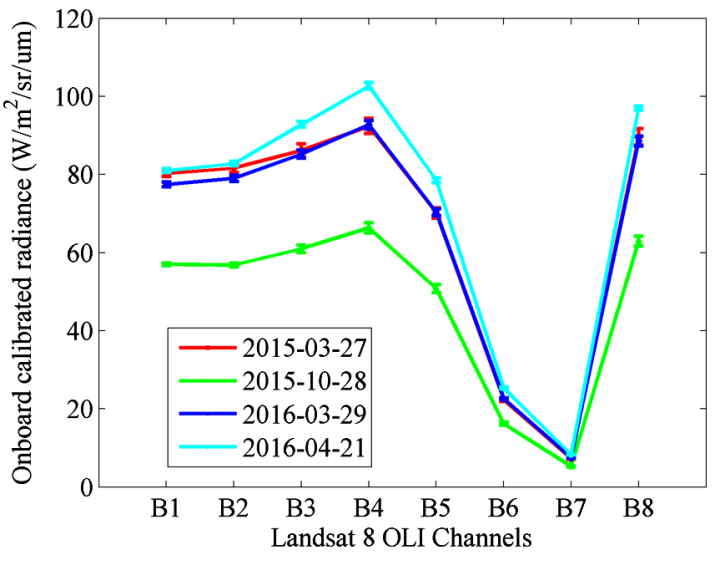

Fig. 3 The average and $1 \delta$ standard deviation of the observed OLI band TOA radiance of the desert target, as calibrated using the onboard calibrator.

radiometric calibration. It should be noted that channel 9 has not been included in our study since it is a cirrus cloud detection channel and is not intended for ground observations.

\subsection{Atmospheric Datasets}

An automatic sun tracking photometer (CE318, CIMEL Electronique, France) was used to measure the direct solar irradiance and sky irradiance on the dates that the Landsat- 8 satellite passed over the Baotou site. The collected data were sent to the Aerosol Robotic Network (AEROSOL) data center to process, since the Baotou site has joined AERONET. The aerosol optical thickness (AOT) values in the 440-, 670-, 870-, and 1020-nm channels were retrieved from the measured direct irradiance of the sun based on the Langley algorithm. The AOT at $550 \mathrm{~nm}$ was then calculated via logarithmic interpolation from the AOT in the 440-, 670-, 870-, and 1020-nm channels. The measurements performed in the 936-nm channel of the solar radiometer were used to calculate the total columnar water vapor (CWV), using a modified version of the Langley algorithm. ${ }^{24}$ Figure 4 shows the main atmospheric parameters of the AOT at $550 \mathrm{~nm}$ and CWV during the field experiment. The linearly interpolated AOT at $550 \mathrm{~nm}$ values at the times when the Landsat- 8 satellite passed over the Baotou site were 0.2828, 0.134, 0.2256, and 0.2703 on March 27, 2015, October 28, 2015, March 29, 2016, and April 21, 2016, respectively, and the corresponding CWV values were $0.6476,0.49,0.4191$, and $0.5518 \mathrm{~g} / \mathrm{cm}^{2}$.

In addition to the atmospheric datasets obtained from the automatic sun tracking photometer CE318, vertical atmospheric profiles of temperature, humidity, and pressure were also collected with a radiosonde balloon at the times of the Landsat- 8 satellite overpasses at our Baotou site on

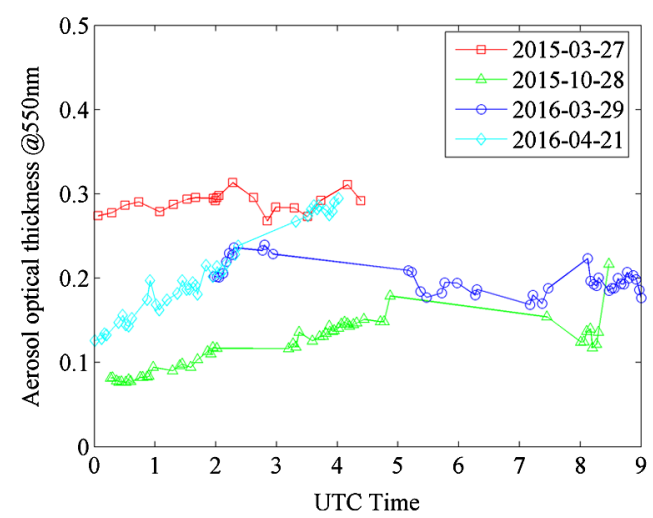

(a)

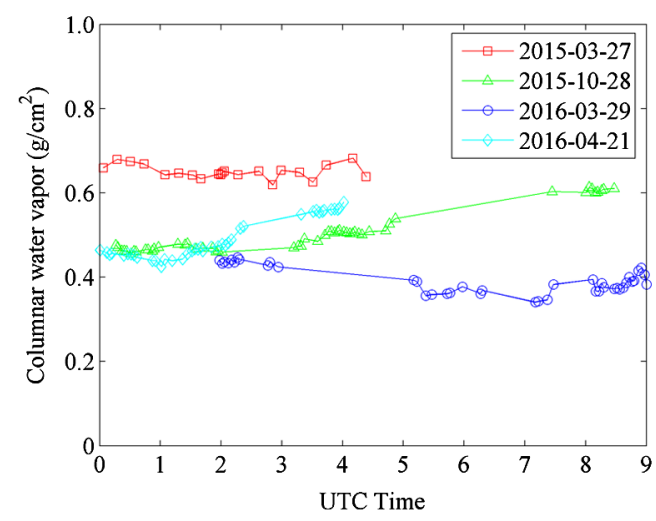

(b)

Fig. 4 The (a) AOT at $550 \mathrm{~nm}$ and (b) CWV atmospheric parameters during the field experiments on March 27, 2015, October 28, 2015, March 29, 2016, and April 21, 2016. 


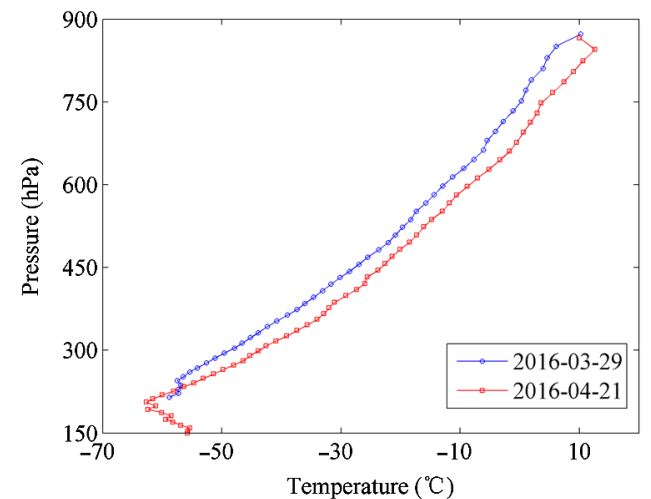

(a)

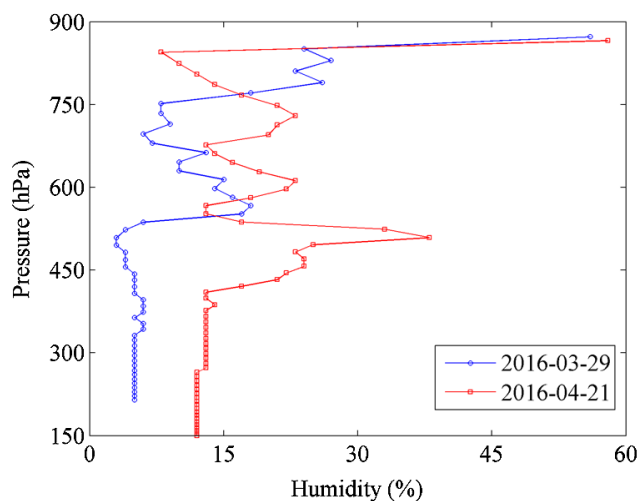

(b)

Fig. 5 The atmospheric vertical profiles collected with radiosonde balloon at the time of the Landsat-8 satellite overpass the Baotou site on March 29, 2016, and April 21, 2016. (a) Pressure versus temperature and (b) pressure versus humidity.

March 29, 2016, and April 21, 2016. The radiosonde balloon collected measurements of temperature, humidity, and pressure every $200 \mathrm{~m}$ vertically, from the ground to $10 \mathrm{~km}$ in height. Figure 5 shows the atmospheric profiles collected on March 29, 2016, and April 21, 2016. These measured atmospheric datasets were used as input to drive the moderate resolution atmospheric transmission (MODTRAN5) radiative transfer code.

\subsection{Ground Reflected Radiance Datasets}

The ground reflected radiance of the desert calibration target was measured using an SVC HR-1024 field portable spectroradiometer (Spectra Vista Corporation, Poughkeepsie, New York). The spectral resolution of the SVC HR-1024 spectrometer is $3.5 \mathrm{~nm}$ from 350 to $1000 \mathrm{~nm}, 9.5 \mathrm{~nm}$ from 1000 to $1850 \mathrm{~nm}$, and $6.5 \mathrm{~nm}$ for the 1850 to $2500-\mathrm{nm}$ spectral range. The SVC HR-1024 spectrometer was radiometrically and spectrally strictly calibrated at the National Institute of Metrology of China. The ground-reflected radiance of the desert target was measured using the SVC HR-1024 spectroradiometer at a height of $\sim 1 \mathrm{~m}$ above the ground at times half an hour before and after the Landsat- 8 satellite passed over the Baotou site on March 27, 2015, October 28, 2015, March 29, 2016, and April 21, 2016. Finally, the ground-reflected radiance of the desert target was averaged with $\sim 120$ samples from all the measurements.

Figure 6 shows the averaged ground reflected radiance of the desert target on March 27, 2015, October 28, 2015, March 29, 2016, and April 21, 2016. Both the averaged ground-

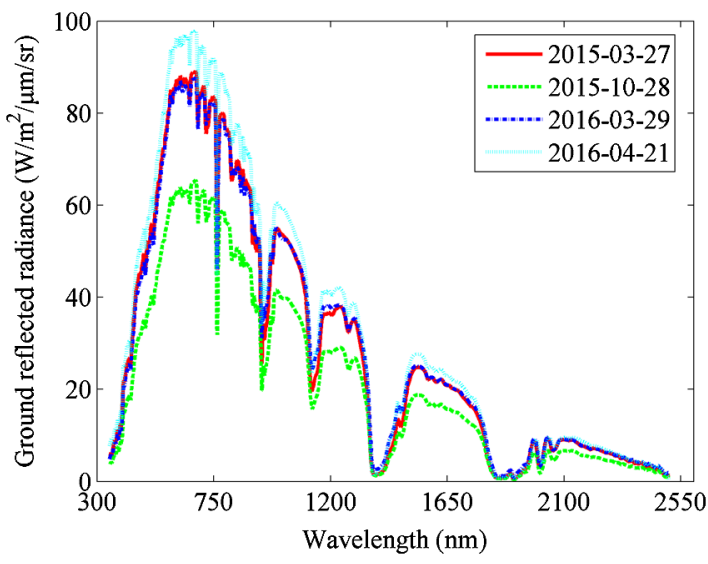

Fig. 6 The averaged ground reflected radiance of the desert target on March 27, 2015, October 28, 2015, March 29, 2016, and April 21, 2016. 
reflected radiance and reflectance are very similar for March 27, 2015 and March 29, 2016, while the averaged ground reflected radiance on April 21, 2016, is larger than the averaged ground reflected radiance on March 27, 2015 and March 29, 2016, which may be due to the solar zenith angles (SZA) change with seasonal variation. The SZA are 43.46, 55.61, 42.29, and $33.37 \mathrm{deg}$ on March 27, 2015, October 28, 2015, March 29, 2016, and April 21, 2016, respectively. It can be found that the SZA are very similar with each other on March 27, 2015, and March 29, 2016, whereas the SZA on October 28, 2015, and April 21, 2016, are very different with the other 2 days. However, the values of the ground-reflected radiance measured on October 28, 2015, are smaller than those measured on the other three days. The decrease in the average groundreflected radiance on October 28, 2015, may be due to the surface humidity at the desert target. The desert target was leveled several days before October 28, 2015, in order to keep the surface uniform and flat, and sand with more moisture under the surface of the desert may have caused the humidity to increase.

\section{Ground Reflected Radiance-Based Vicarious Radiometric Calibration/Validation Approach}

As is well known, the first step of vicarious radiometric calibration/validation is to predict the at-sensor radiance, which is usually determined using a radiative transfer code with in situmeasured surface reflected information and atmospheric characteristics. According to the radiative transfer equation, the at-sensor radiance can be described using Eq. (1) when the surface is assumed to be uniform and Lambertian under a horizontally homogeneous atmosphere: ${ }^{25}$

$$
L_{\mathrm{TOA}}(\lambda)=L_{\mathrm{path}}(\lambda)+\frac{\rho(\lambda)}{\pi[1-\rho(\lambda) S(\lambda)]} \mu_{s} E_{\mathrm{s}}(\lambda) \tau\left(\mu_{s}, \lambda\right) \tau\left(\mu_{v}, \lambda\right),
$$

where $L_{\mathrm{TOA}}(\lambda)$ is the at-sensor spectral TOA radiance with unit $\mathrm{W} / \mathrm{m}^{2} / \mathrm{sr} / \mu \mathrm{m} ; L_{\mathrm{path}}(\lambda)$ is the path radiance with unit $\mathrm{W} / \mathrm{m}^{2} / \mathrm{sr} / \mu \mathrm{m} ; \rho(\lambda)$ is the surface reflectance; $S(\lambda)$ is the atmospheric spherical albedo; $E_{\mathrm{s}}(\lambda)$ is the solar irradiance with unit $\mathrm{W} / \mathrm{m}^{2} / \mu \mathrm{m} ; \tau\left(\mu_{s}, \lambda\right)$ is the total transmittance from the sun to the surface; $\tau\left(\mu_{v}, \lambda\right)$ is the total transmittance from the surface to the sensor; $\mu_{s}$ and $\mu_{v}$ are the cosine values of the solar and viewing zenith angles.

As we can see from Eq. (1), the at-sensor radiance can be determined using the MODTRAN5 radiative transfer code once the surface reflectance and atmospheric conditions are known. Therefore, the measurement of surface reflectance values over the calibration site is a necessary prerequisite to predict the at-sensor radiance, and this is also the foundation of the reflectance-based vicarious radiometric calibration approach. However, the reflectance-based approach is reported that there are large uncertainties of the calibration target reflectance measurements. ${ }^{26}$ It has also been reported that there is a systematic error in the reflectancebased approach, due to the calculation of aerosol properties. ${ }^{27}$ Therefore, a radiance-based approach was developed to overcome the drawbacks by measuring the radiance of the calibration targets using strictly calibrated spectrometer at high elevation like an airborne platform. ${ }^{21}$ However, it is impractical to measure the calibration target radiance always at an airborne platform due to the high cost and difficulty in operation.

To overcome the abovementioned problems of the reflectance-based and airborne radiancebased approach, a ground reflected radiance-based approach is suggested in this study for predicting the at-sensor radiance. The ground reflected radiance-based approach supposing that the reflected radiance of the calibration target as expressed by Eq. (2) can be measured directly by a strictly radiometrically calibrated spectrometer:

$$
L_{g}(\lambda)=\frac{\rho(\lambda) \cdot E_{\mathrm{s}}(\lambda) \cdot \tau\left(\mu_{s}, \lambda\right)}{\pi},
$$

where $L_{g}(\lambda)$ is the reflected radiance of the calibration target with unit $\mathrm{W} / \mathrm{m}^{2} / \mathrm{sr} / \mu \mathrm{m}$. Then, the TOA radiance can be predicted by Eq. (3) after rearranging Eq. (1) with Eq. (2): 


$$
L_{\mathrm{TOA}}(\lambda)=L_{\mathrm{path}}(\lambda)+\frac{L_{g}(\lambda) \cdot \tau\left(\mu_{v}, \lambda\right)}{1-\frac{\pi L_{g}(\lambda)}{\mu_{s} E_{s}(\lambda) \tau\left(\mu_{s}, \lambda\right)} S(\lambda)} .
$$

The multiple scatter between atmosphere, calibration targets, and adjacent pixels can be neglected due to the large size and homogeneous of the calibration site. Then, Eq. (3) can be simplified by Eq. (4):

$$
L_{\mathrm{TOA}}(\lambda)=L_{\mathrm{path}}(\lambda)+L_{g}(\lambda) \cdot \tau\left(\mu_{v}, \lambda\right) .
$$

It can be seen from Eq. (4) that the reflected radiance of the calibration targets, the path radiance, and the total transmittance in the view direction are the primary unknowns involved in predicting the spectral TOA radiance. The ground-reflected radiance was measured by a strictly radiometrically calibrated spectrometer at the time when the Landsat- 8 satellite passed over the Baotou site. In addition, the path radiance and total transmittance in the view direction were simulated with the MODTRAN5 radiative transfer code, using the atmospheric data from the Baotou AERONET station. Compared with the traditional reflectance-based approach in which uncertainties in the two-way atmospheric path (namely the solar direction and sensor view direction) and reflectance measurement exist has to be accounted for by the radiative transfer code, only one-way path in the sensor view direction should be considered according to Eq. (4). Furthermore, the main error source of the ground reflected radiance-based approach is the uncertainty in the calibration of the spectrometer. Compared with the airborne radiance-based approach, the ground reflected radiance-based approach is more convenient to operate and the cost is low.

Based on the predicted spectral TOA radiance, the band TOA radiance of the OLI sensor can then be calculated by convoluting the spectral TOA radiance with the spectral response function of the corresponding channels, as described by Eq. (5):

$$
L_{\mathrm{TOA}, i}=\int_{\lambda_{1}}^{\lambda_{2}} R_{i}(\lambda) \cdot L_{\mathrm{TOA}}(\lambda) \mathrm{d} \lambda / \int_{\lambda_{1}}^{\lambda_{2}} R_{i}(\lambda) \mathrm{d} \lambda,
$$

where $L_{\mathrm{TOA}, i}$ is the OLI TOA radiance in band $i ; R_{i}(\lambda)$ is the spectral response function of the corresponding bands; $L_{\mathrm{TOA}}(\lambda)$ is the continuous TOA spectral radiance; and $\lambda_{1}$ and $\lambda_{2}$ are the lower and upper wavelength of the spectral range in band $i$, respectively.

\section{Results and Discussion}

\subsection{Vicarious Radiometric Calibration/Validation Results of Landsat-8 OLI}

As described in Sec. 3, the ground reflected radiance-based approach was applied to all the datasets collected during the field campaign. A ratio expressed by Eq. (6) was used to quantitatively evaluate the differences between the predicted and observed TOA radiance values:

$$
\text { Ratio }=\frac{\text { Predicted }}{\text { Observed }}
$$

where predicted stands for the OLI band TOA radiance predicted with the ground reflected radiance-based approach, based on the ground measurements at the time when the Landsat- 8 satellite passed over our calibration site, and observed stands for the OLI band TOA radiance calculated with the radiometric calibration coefficients provided with the Landsat/OLI data. Figure 7 shows the ratio between the predicted and observed OLI band TOA radiance values using the ground reflected radiance-based method.

It can be seen from Fig. 7 that the trend of the ratios between the predicted and observed results is very similar over the eight channels. The relative differences between the predicted and observed OLI band TOA radiance values are less than $5 \%$, and the mean relative differences for the eight channels are less than 2\% on March 27, 2015, and March 29, 2016. However, the predicted OLI band TOA radiance values on October 28, 2015, are more similar to the observed 


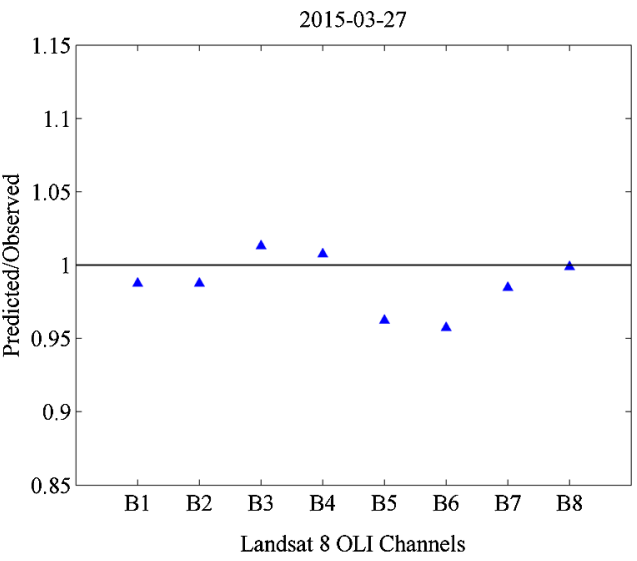

(a)

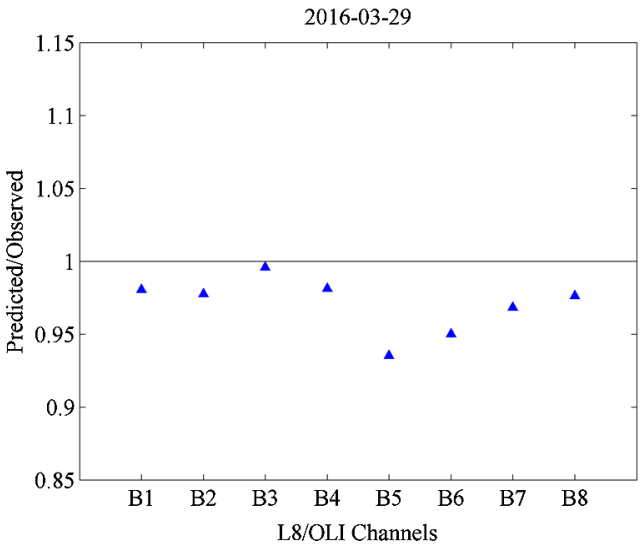

(c)

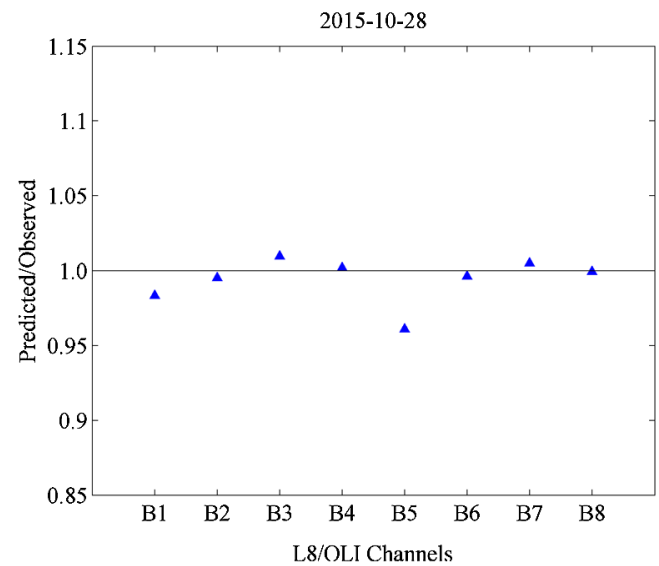

(b)

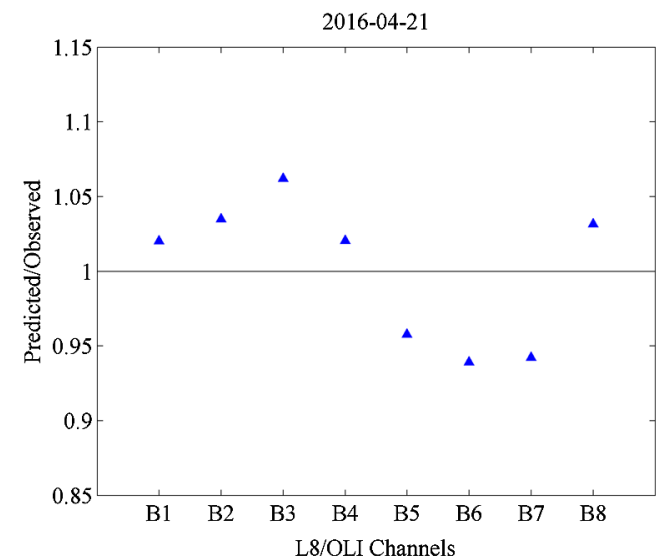

(d)

Fig. 7 Relative differences between the predicted and observed OLI band TOA radiance values at times when the Landsat-8 satellite passed over the Baotou site on (a) March 27, 2015, (b) October 28, 2015, (c) March 29, 2016, and (d) April 21, 2016, respectively.

values, with mean relative differences of less than $1 \%$ for the eight OLI channels. The improved atmospheric conditions that occurred on October 28, 2015, may have contributed to the better results obtained on that day, compared to the other days. It can be seen from Fig. 4 that the measured AOT at $550 \mathrm{~nm}$ and CWV on October 28, 2015, are only 0.134 and $0.49 \mathrm{~g} / \mathrm{cm}^{2}$, respectively. In addition, the measured atmospheric parameters of AOT at $550 \mathrm{~nm}$ and CWV are more stable before and after the Landsat- 8 satellite passed over the Baotou site than on the other days. It also can be seen from Fig. 7 that the relative differences at band 5, band 6, and band 7 are larger than the other bands, which were explained due to the low signal in these bands covering the short-wave infrared wavelength. ${ }^{2}$

It is interesting to note that there is an obvious difference between the results when comparing the predicted to the observed OLI band TOA radiance values on April 21, 2016. The residuals from the mean radiometric rescaling coefficients of the OLI sensor provided in the product metadata file (MTL file) are plotted in Fig. 8. It can be seen from Fig. 8 that the residuals in the gains and biases on March 27, 2015, October 28, 2015, and March 29, 2016, have similar trends and are inconsistent with each other, whereas the residuals associated with both the radiometric rescaling gains and biases on April 21,2016, display apparent differences compared to the residuals associated with the radiometric rescaling gains and bias from the preceding three days. In addition, these differences in the gains and the biases may lead to inconsistencies between the observed and predicted OLI band TOA radiance values.

Figure 9 shows the ratios between the predicted and observed OLI band TOA radiance values obtained by averaging the results from the four field campaign calibrations. The relative errors of the eight OLI bands are within 5\% when compared to the predicted with the onboard observed 


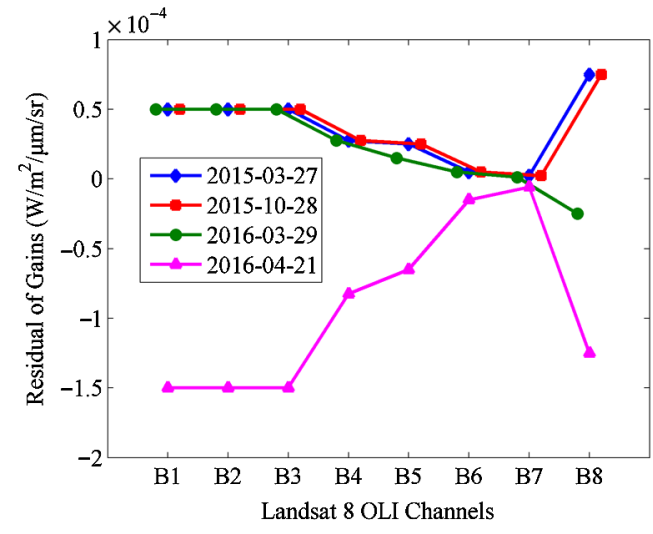

(a)

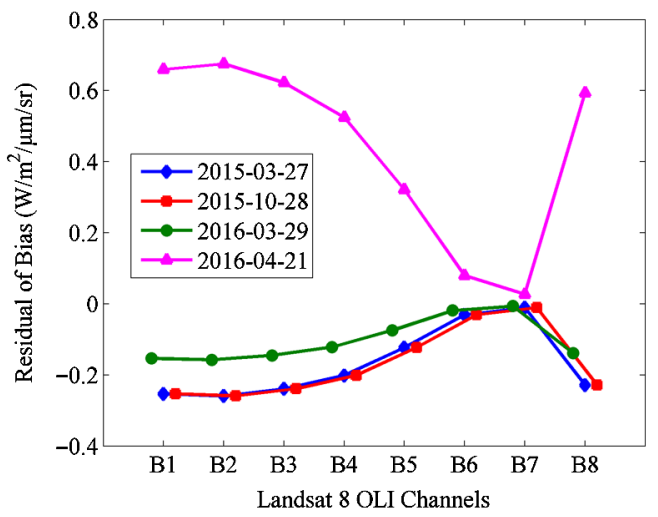

(b)

Fig. 8 The residuals associated with the mean radiometric rescaling coefficients of the OLI sensor provided in the product metadata file (MTL file) for the four days. (a) Residuals associated with gains and (b) residuals associated with biases.

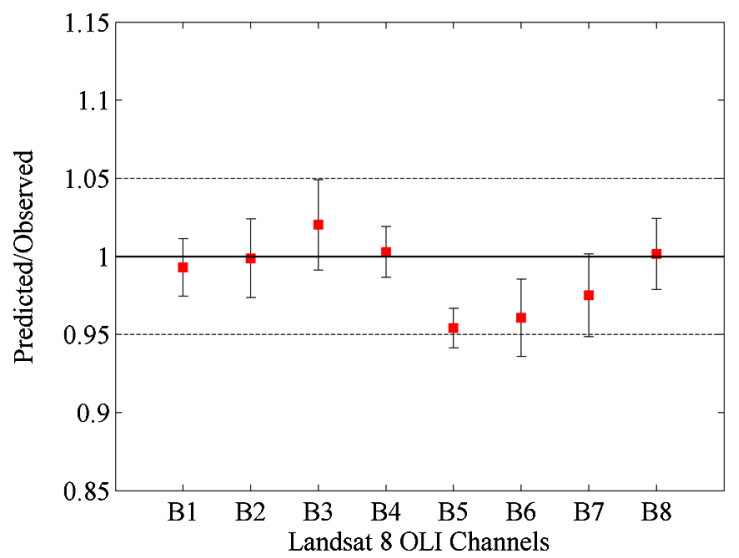

Fig. 9 The mean ratio between predicted and observed OLI band TOA radiance for the four days field campaign calibration and the error bar is the $1 \delta$ standard deviation of the ratio for the four days.

OLI band TOA radiance values. The error bars shown in Fig. 9 are the $1 \sigma$ standard deviation of the ratio between the predicted and observed OLI band TOA radiance values obtained during the four-field campaign calibrations. The $1 \delta$ standard deviations are $0.018,0.025,0.029,0.016$, $0.013,0.025,0.027$, and 0.023 for the eight OLI channels, respectively. Figure 9 demonstrates that the onboard radiometric calibration results show good consistency with our vicarious radiometric calibration results with a mean relative error less than 5\%, which shows similar results by compared with the previous reported Landsat-8 OLI vicarious radiometric calibration results. ${ }^{2}$

\subsection{Cross-Radiometric Comparison Results between Landsat-8 OLI and Terra MODIS Senor}

As a cross-radiometric comparison, the calibration from well-calibrated sensors, such as MODIS, MISR, and VIIRS (ETM+ in this case), should be used to cross-calibrate the Landsat-8 OLI sensor. In this study, the well radiometrically calibrated MOD02HKM product acquired by Terra MODIS sensor is referenced for comparison to the Landsat- 8 OLI sensor. The Landsat- 8 OLI dataset were resampled to the same $500-\mathrm{m}$ spatial resolution as MOD02HKM product. For best results, a larger uniform desert area near the BAOTOU site was imaged nearly by the two sensors on March 27, 2015, October 28, 2015, March 29, 2016, and April 21, 2016. The overpass UTC time for Terra MODIS and Landsat-8 OLI is nearly at 02:50 and 


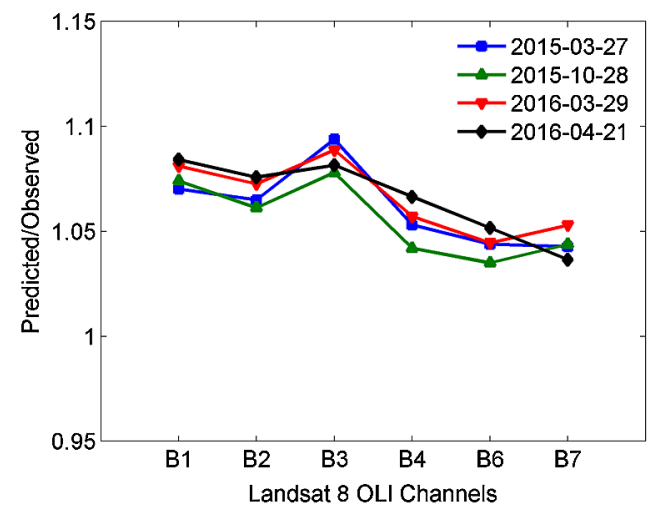

(a)

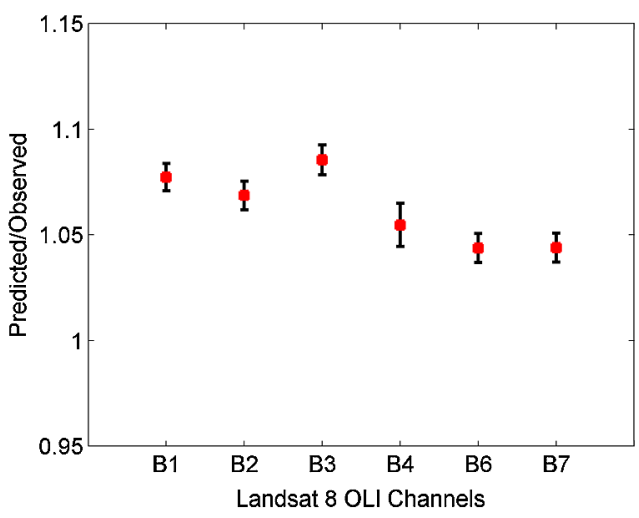

(b)

Fig. 10 (a) The ratios between cross calibrated (predicted) and onboard calibrated (observed) TOA radiance of the Landsat-8 OLI sensor. (b) The mean ratio between cross calibrated and observed calibrated TOA radiance of the Landsat-8 OLI sensor and the $1 \delta$ standard deviation of the ratio for the four calibration days.

03:20, respectively. More than 100 desert pixels were subtracted from the Terra MODIS MOD02HKM and Landsat-8 OLI products. Then, the digital counts were averaged from the subtracted pixels and converted to radiance using the onboard calibration coefficients.

By comparing the spectral response function between Terra MODIS and Landsat-8 OLI senor, only six channels covering the blue, green, red, NIR, SWIR1, and SWIR2 were cross compared. First, the TOA radiance of the selected desert corresponding to Terra MODIS and Landsat-8 OLI sensor spectral response function was simulated from the radiative transfer code MODTRAN 5, assuming that the sun zenith angle, viewing angle, and atmospheric parameters changed slightly. Then, the spectral correction factor between Terra MODIS and Landsat- 8 OLI sensor radiance conversation was calculated based on predicted datasets. After then, the Landsat-8 OLI TOA radiance of the desert was predicted based on the spectral correction factor and reference Terra MODIS onboard well radiometrically calibrated radiance. Finally, the predicted value based on reference Terra MODIS sensor and Landsat-8 OLI onboard calibrated radiance was compared.

Figure 10 shows the ratio between cross calibrated and onboard calibrated TOA radiance of the Landsat-8 OLI sensor corresponding to the four field calibration campaign days. It can be seen from the cross comparisons that there are similar trends for the four calibration days over the five channels. However, the relative errors of the cross validation results are larger than the previous shown vicarious radiometric validation results. The mean relative errors of the crosscalibrated Landsat OLI sensor channels for the four days are $7.74 \%, 6.87 \%, 8.56 \%, 5.47 \%$, $4.38 \%$, and $4.40 \%$, respectively. These relative errors may be due to the registration of two sensor observed images, the atmospheric, sun and sensor viewing angles changes between overpassed of the sensors. ${ }^{28}$ The spectral correction factors calculated based on the radiative transfer code with Terra MODIS and Landsat-8 OLI sensor spectral response function may also cause the cross-calibration and validation errors. ${ }^{29}$ It also can be noted from Fig. 10 that the relative errors in the VNIR channels are larger than the shortwave channels, which may be due to the aerosol atmospheric effects in the VNIR channels being more than apparent than shortwave channels.

\subsection{Uncertainty Analysis}

In practice, various uncertainties in the observed data, data processing, and selected vicarious radiometric calibration method are inevitable, which may consequently affect the final accuracy of the vicarious radiometric calibration. Therefore, it is crucial to determine the uncertainty associated with various sources that may contribute to the uncertainty of the predicted TOA radiance using the ground reflected radiance-based and traditional reflectance-based approaches. From the description of the ground reflected radiance-based method given earlier in Sec. 2, it can be seen that there are several potential sources of uncertainty, including surface characteristics, atmospheric characteristics, and the selected calibration model. 
In terms of the uncertainties of the surface characteristics, three factors should be taken into consideration: (1) the calibration uncertainty of the SVC HR-1024 spectrometer in the laboratory, which has an uncertainty of $4 \%$; (2) the uncertainty of the measured ground reflected radiance due to the uniformity of the desert target and the stability of the measurements, which can be estimated from the ground reflected radiance measured using the SVC HR-1024 spectrometer; and (3) the bidirectional reflectance distribution function (BRDF) characteristics of the desert target, which have uncertainties less than $1.5 \%$ when the viewing zenith angle is less than $10 \mathrm{deg}$, according to analysis of the bidirectional reflectance measurements. With regard to the atmospheric characteristics, the uncertainties of the estimated AOT at $550 \mathrm{~nm}$ and the CWV from the data obtained using the CE318 photometer are 5.5\% and 10\%, as reported by the AERONET. The factors of the chosen calibration model refer to the uncertainties of the ground reflected radiance-based model, the radiative transfer code MODTRAN5, and the solar irradiance model. The maximum uncertainty of the ground reflected radiance-based method used in the study is estimated to be less than $1 \%$. In addition, the uncertainty associated with the radiative transfer code MODTRAN5 is $2 \%$ and 0.005 for radiance and transmittance predictions, respectively. ${ }^{30}$ The uncertainty associated with the solar irradiance model is about $1 \%$ according to the summary by Czapla-Myers et al. ${ }^{2}$ based on Thuillier et al. ${ }^{31,32}$ research.

The TOA radiance uncertainties due to the uncertainty of the surface, atmospheric characteristics, and the ground reflected radiance-based model and MODTRAN5 model, solar irradiance model uncertainties were estimated by adding the uncertainties using a simulation method. First, the TOA radiance without uncertainties was simulated with MODTRAN. Then, the TOA radiance by adding the uncertainty error was simulated with MODTRAN. Finally, the relative error of the TOA radiance due to the uncertainty sources (e.g., the AOT) was calculated according to Eq. (8). However, the uncertainties associated with the solar irradiance model would result in uncertainties in the TOA radiance of less than $1 \%$, since the source of uncertainty associated with solar irradiance is assumed to be independent. ${ }^{23}$ Finally, the overall uncertainty of the OLI TOA radiance is calculated using Eq. (7), taking into account all the relevant factors:

$$
U_{\mathrm{AOT}}=\frac{L_{i}(\mathrm{AOT})-L_{i}(\mathrm{AOT}+\Delta)}{L_{i}(\mathrm{AOT})}
$$

where $U_{\mathrm{AOT}}$ is the uncertainty error of TOA radiance due to the measured AOT uncertainty error. $L_{i}(\mathrm{AOT})$ is the TOA radiance simulated with measured AOT, $L_{i}(\mathrm{AOT}+\Delta)$ is the TOA radiance by adding the uncertainty error to the measured AOT (the same as to other uncertainty sources):

$$
U_{\mathrm{TOA}}=\sqrt{U_{\text {cali }}^{2}+U_{\text {meas }}^{2}+U_{\mathrm{BRDF}}^{2}+U_{\mathrm{AOT}}^{2}+U_{\mathrm{CWV}}^{2}+U_{\mathrm{MODEL}}^{2}+U_{\mathrm{MODTRAN}}^{2}+U_{\mathrm{ESUN}}^{2}},
$$

where $U_{\mathrm{TOA}}$ is the overall uncertainty of the TOA radiance results from all the uncertainty sources; $U_{\text {cali }}, U_{\text {meas }}, U_{\mathrm{BRDF}}, U_{\mathrm{AOT}}, U_{\mathrm{CWV}}, U_{\mathrm{MODEL}}, U_{\mathrm{MODTRAN}}$, and $U_{\mathrm{ESUN}}$ are the uncertainties of the TOA radiance that result from the uncertainty of the spectrometer calibration, the uniformity and stability of the desert target, the BRDF of the desert target, the AOT at $550 \mathrm{~nm}$, the CWV, the ground reflected radiance-based model, the MODTRAN5 model, and the solar irradiance model, respectively.

The overall uncertainties of the OLI TOA radiance in function of the spectral bands, as determined from the sources of uncertainty discussed above, are listed in Table 2. It can be seen from Table 2 that the averaged overall uncertainties of the OLI TOA radiance are less than $2.94 \%$, $3.45 \%, 4.26 \%, 4.62 \%, 4.76 \%, 4.89 \%, 4.93 \%$, and $4.35 \%$ for the eight channels, respectively. It can be concluded that the main uncertainty sources that contribute to the uncertainties in the OLI TOA radiance are the laboratory radiometric calibration of the spectrometer and the measured reflected radiance, due to the uniformity and stability of the measurements of the desert target. However, the uncertainties in the TOA radiance are less than $0.5 \%$ and $0.02 \%$, due to the uncertainty sources associated with the AOT at $550 \mathrm{~nm}$ and CWV atmospheric parameters. Therefore, accurate measurement of the ground reflected radiance is very important to ensure the accuracy of the vicarious radiometric calibration. 
Table 2 The uncertainty analysis results of the ground reflected radiance-based approach.

\begin{tabular}{lccccccccc}
\hline \hline & & \multicolumn{7}{c}{ TOA radiance uncertainty (\%) } \\
\cline { 3 - 9 } Uncertainty sources & $\begin{array}{c}\text { Sources } \\
\text { uncertainty (\%) }\end{array}$ & B1 & B2 & B3 & B4 & B5 & B6 & B7 & B8 \\
\hline Spectrometer calibration & 4.00 & 1.60 & 2.19 & 3.10 & 3.55 & 3.83 & 3.97 & 3.99 & 3.23 \\
Measured reflected radiance & 3.00 & 1.54 & 1.97 & 2.45 & 2.57 & 2.46 & 2.35 & 2.51 & 2.48 \\
BRDF characteristics & 1.50 & 0.53 & 0.69 & 0.80 & 0.78 & 0.78 & 0.72 & 0.82 & 0.79 \\
Aerosol optical thickness & 5.50 & 0.60 & 0.52 & 0.30 & 0.15 & 0.05 & 0.02 & 0.01 & 0.25 \\
CWV & 10.00 & 0.00 & 0.00 & 0.01 & 0.01 & 0.00 & 0.01 & 0.06 & 0.01 \\
Radiance-based model & 1.00 & 0.07 & 0.04 & 0.02 & 0.07 & 0.08 & 0.88 & 0.45 & 0.04 \\
MODTRAN5 model & 2.00 & 1.43 & 1.21 & 0.88 & 0.70 & 0.58 & 0.53 & 0.52 & 0.83 \\
Solar irradiance model & 1.00 & 1.00 & 1.00 & 1.00 & 1.00 & 1.00 & 1.00 & 1.00 & 1.00 \\
Overall uncertainty & - & 2.94 & 3.45 & 4.26 & 4.62 & 4.76 & 4.89 & 4.93 & 4.35 \\
\hline \hline
\end{tabular}

\section{Conclusions}

In this paper, a ground reflected radiance-based approach, based on the traditional reflectancebased and airborne radiance-based approaches, has been used to carry out vicarious radiometric calibration of the Landsat-8 OLI sensor. Compared to the reflectance-based approach, the advantages of the ground radiance-based approach used in this study can be briefly summarized as the following. First, the ground reflected radiance-based approach can overcome the measurement uncertainty associated with the calibration site surface reflectance characteristics when comparing with the traditional reflectance-based approach, for example, the degradation and contamination of the reference panel in the field environment. In addition, the ground reflected radiancebased approach can avoid the drawback that the view angles might be different when measuring the reflected radiance of target and reference panel. Furthermore, only the total transmittance in the view direction should be calculated for the ground reflected radiance-based approach, instead of the total transmittance at both the solar and view directions that are used in the reflectancebased approach, which may minimize the uncertainty associated with the chosen default atmospheric model and aerosol type. Moreover, it is also very convenient and operationally feasible to carry out the ground reflected radiance-based approach, compared with airborne radiance-based and irradiance-based approaches. However, it should be noted that the spectrometer used to measure the ground reflected radiance should be strictly radiometrically and spectrally calibrated before the vicarious radiometric calibration is performed since the measured ground reflected radiance is the main source of uncertainty source, as described in the uncertainty analysis presented in Sec. 4. In addition, the vicarious absolute radiometric calibration coefficients of the OLI have not been given in this study, since only one calibration site with middle surface reflected characteristics, which may affect the accuracy of the calibration coefficients when fitting the predicted OLI band TOA radiance with the observed digital values. The calibration site at Ulansuhai Lake, which has low reflected characteristics and lies $\sim 50 \mathrm{~km}$ away from the Baotou site, will be considered as an additional calibration site. The results will be combined with those obtained at the Baotou site to determine the vicarious absolute radiometric calibration coefficients.

With the ground reflected radiance-based approach, the radiometric performance has been evaluated based on four vicarious calibration datasets acquired at the Baotou site from March 2015 to April 2016. The vicarious absolute radiometric calibration results of the ground reflected radiance-based approach show a smaller bias when comparing the predicted and observed OLI band TOA radiance values. The relative errors of the eight OLI bands are within 5\% when compared with the onboard observed OLI band TOA radiance values. The results also show that the average $1 \delta$ standard deviation of the four vicarious calibration datasets for the four vicarious calibration datasets is less than 0.025 . Besides, a well radiometric calibrated Terra MODIS 
sensor was used to cross validate the Landsat- 8 OLI onboard radiometric calibration results, which also shows a good consistency between predicted and observed Landsat-8 OLI TOA radiance and the relative errors are within $10 \%$. Both of the vicarious and cross radiometrically validation suggest that the OLI onboard the Landsat- 8 satellite displays a stable radiometric performance for the four calibration/validation days, except for slight rescaling factor changes on April 21, 2016, which may be due to the variations in Earth-Sun distance. The uncertainty of the predicted OLI radiance values using the ground reflected radiance-based approach has been analyzed by considering the sources of uncertainty, including surface characteristics, atmospheric characteristics, and the chosen calibration model. The results indicate that the overall uncertainty of the predicted OLI radiance values is less than $4.142 \%, 4.272 \%, 4.144 \%$, and 4.290\% on March 27, 2015, October 28, 2015, March 29, 2016, and April 21, 2016, respectively.

Overall, the results of the preliminary vicarious radiometric calibration obtained using the ground reflected radiance-based approach have been demonstrated that the Landsat-8 OLI sensor shows good radiometric performance over the calibration period in this study. Carrying out long-term ground-based observations of surface characteristics and atmospheric conditions at times when the Landsat- 8 satellite passes over our Baotou site and a more detailed analysis of the uncertainties contributed by different sources will be our main work in the near future.

\section{Disclosures}

The authors declare no conflict of interest.

\section{Acknowledgments}

This work is partially supported by the National Natural Science Foundation of China (ID: 41601398) and the National High Technology Research and Development Program of China (ID: 2014AA123201). The authors would also like to thank the Institute of Remote Sensing and Digital Earth (RADI) for providing us with the Landsat-8 data.

\section{References}

1. J. Barsi et al., "Landsat-8 thermal infrared sensor (TIRS) vicarious radiometric calibration," Remote Sens. 6, 11607-11626 (2014).

2. J. Czapla-Myers et al., "The ground-based absolute radiometric calibration of OLI," Remote Sens. 7, 600-626 (2015).

3. E. Knight and G. Kvaran, "Landsat-8 operational land imager design, characterization, and performance," Remote Sens. 6, 10286-10305 (2014).

4. B. Markham et al., "Landsat- 8 operational land imager radiometric calibration and stability," Remote Sens. 6, 12275-12308 (2014).

5. C. Dymond, D. Mladenoff, and V. Radeloff, "Phenological differences in tasseled cap indices improve deciduous forest classification," Remote Sens. Environ. 80, 460-472 (2002).

6. L. Lymburner, P. Beggs, and C. Jacobson, "Estimation of canopy-average surface-specific leaf area using Landsat TM data," Photogramm. Eng. Remote Sens. 66(2), 183-191 (2000).

7. M. Steininger, "Satellite estimation of tropical secondary forest aboveground biomass: data from Brazil and Bolivia," Int. J. Remote Sens. 21, 1139-1157 (2000).

8. J. Price, "Radiometric calibration of satellite near infrared: history and outlook," Remote Sens. Environ. 22, 3-9 (1987).

9. G. Chander, B. Markham, and D. Helder, "Summary of current radiometric calibration coefficients for Landsat MSS, TM, ETM+, and EO-1 ALI sensors," Remote Sens. Environ. 113, 893-903 (2009).

10. L. Paolini et al., "Radiometric correction effects in Landsat multi-date/multi-sensor change detection studies," Int. J. Remote Sens. 27(4), 685-704 (2006).

11. D. Helder et al., "Absolute radiometric calibration of Landsat using a pseudo invariant calibration site," IEEE Trans. Geosci. Remote Sens. 51(3), 1360-1369 (2013).

12. K. Thome et al., "Radiometric calibration of Landsat," Photogramm. Eng. Remote Sens. 63(7), 853-858 (1997). 
13. K. Thome et al., "Landsat-5 TM and Landsat-7 ETM+ absolute radiometric calibration using the reflectance-based method," IEEE Trans. Geosci. Remote Sens. 42(12), 27772785 (2004).

14. J. Horan, D. Schwartz, and J. Love, "Partial performance degradation of a remote sensor in a space environment, and some probable causes," Appl. Opt. 13(5), 1230-1237 (1974).

15. G. Chander et al., "Landsat-5 TM reflective-band absolute radiometric calibration," IEEE Trans. Geosci. Remote Sens. 42(12), 2747-2760 (2004).

16. G. Chander, B. Markham, and J. Barsi, "Revised Landsat-5 thematic mapper radiometric calibration," IEEE Geosci. Remote Sens. 4(3), 490-494 (2007).

17. J. Barsi et al., "Radiometric calibration status of Landsat-7 and Landsat-5," Proc. SPIE 6744, 67441F (2007).

18. B. Markham et al., "Landsat-7 ETM+ on-orbit reflective-band radiometric stability and absolute calibration," IEEE Trans. Geosci. Remote Sens. 42(12), 2810-2820 (2004).

19. B. Markham et al., "Landsat-7 enhanced thematic mapper plus in-flight radiometric calibration," in IEEE Int. Geoscience and Remote Sensing Symp. (IGARSS), Vol. 2, pp. 1273-1275 (1996).

20. P. Slater et al., "Reflectance- and radiance-based methods for the in-flight absolute calibration of multispectral sensors," Remote Sens. Environ. 22, 11-37 (1987).

21. P. Slater et al., "Vicarious radiometric calibrations of EOS sensors," J. Atmos. Oceanic Technol. 13, 349-359 (1996).

22. S. Biggar, R. Santer, and P. Slater, "Irradiance-based calibration of imaging sensors," in IEEE Int. Geoscience and Remote Sensing Symp. (IGARSS), Vol. 1, pp. 507-510 (1990).

23. K. Thome, "Absolute radiometric calibration of Landsat 7 ETM+ using the reflectancebased method," Remote Sens. Environ. 78, 27-38 (2001).

24. J. A. Reagan et al., "Water vapor measurements in the 0.94 micron absorption band: calibration, measurements and data applications," in IEEE Int. Geoscience and Remote Sensing Symp. (IGARSS), pp. 18-21 (1987).

25. S. Liang, H. Fang, and M. Chen, "Atmospheric correction of Landsat ETM+ land surface imagery-part I: methods," IEEE Trans. Geosci. Remote Sens. 39(11), 2490-2498 (2001).

26. X. Gu, G. Guyot, and M. Verbrugghe, "Evaluation of measurement errors in ground surface reflectance for satellite calibration," Int. J. Remote Sens. 13(14), 2531-2546 (1992).

27. Z. Chen et al., "Vicarious calibration of Beijing-1 multispectral imagers," Remote Sens. 6, 1432-1450 (2014).

28. J. T. Kurtis, F. B. Stuart, and W. Wit, "Cross comparison of EO-1 sensors and other earth resources sensors to Landsat-7 ETM+ using railroad valley playa," IEEE Trans. Geosci. Remote Sens. 41(6), 1180-1188 (2003).

29. P. M. Teillet, B. L. Markham, and R. I. Richard, "Landsat cross-calibration based on near simultaneous imaging of common ground targets," Remote Sens. Environ. 102, 264-270 (2006).

30. A. Berk et al., MODTRAN5 5.2.1 User's Manual, Spectral Sciences Inc., Air Force Research Laboratory, Burlington, Massachusetts and Hanscom AFB, Massachusetts (2011).

31. G. Thuillier et al., "The visible solar spectral irradiance from 350 to $850 \mathrm{~nm}$ as measured by the SOLSPEC spectrometer during the ATLAS I Mission," in Solar Electromagnetic Radiation Study for Solar Cycle 22, J. M. Pap, C. Fröhlich, and R. K. Ulrich, Eds., pp. 41-61, Springer, Berlin (1998).

32. G. Thuillier et al., "The solar spectral irradiance from 200 to $2400 \mathrm{~nm}$ as measured by the SOLSPEC spectrometer from the Atlas and Eureca Missions," Sol. Phys. 214, 1-22 (2003).

Yaokai Liu is currently pursuing his $\mathrm{PhD}$ degree in remote sensing science at University of Chinese Academy of Sciences, Beijing, China. His research interests include field campaign of remote sensing, spectral and radiometric calibration of optical sensors, hyperspectral data processing and analysis, retrieval of vegetation fractional cover, aerosol optical thickness, and land surface reflectance from remote sensing data.

Biographies for the other authors are not available. 